\title{
Possible heliogeophysical effects on human physiological state
}

\author{
Svetla Dimitrova \\ Solar-Terrestrial Influences Laboratory, Bulgarian Academy of Sciences, \\ Acad. G. Bonchev Str. Bl. 3 Sofia 1113 Bulgaria \\ email: svetla_stil@abv.bg
}

\begin{abstract}
A group of 86 healthy volunteers was examined in periods of high solar and geomagnetic activity. In this study hourly Dst-index values and hourly data about intensity of cosmic rays were used. Results revealed statistically significant increments for the mean systolic and diastolic blood pressure, pulse pressure and subjective psycho-physiological complaints of the group with geomagnetic activity increase and cosmic rays intensity decrease.
\end{abstract}

Keywords. Cosmic rays, geomagnetic activity, blood pressure, subjective complaints

\section{Introduction}

Space weather is often defined as conditions on the Sun and in the solar wind, magnetosphere, ionosphere and thermosphere that can influence the performance and reliability of space-borne and ground-based technological systems and can endanger human life or health. A variety of physical phenomena are associated with space weather, including cosmic ray $(\mathrm{CR})$ intensity variations and geomagnetic storms. Galactic CRs experience significant variation in response to passing solar wind disturbances such as interplanetary coronal mass ejections (ICMEs) and their accompanying shocks. Arriving at Earth, ICMEs compress the magnetosphere, intensify the magnetosphere currents thus leading to a significant depletion of CR intensity (CRI) and producing geomagnetic storms. Some studies revealed significant effects on myocardial infarctions, brain strokes, and traffic accidents on the days of geomagnetic field (GMF) disturbances accompanied with CRI decreases (Villoresi et al. 1995; Ptitsina et al. 1998; Dorman 2005).

\section{Material and methods}

Data were obtained in 86 healthy volunteers in Sofia on working days in autumn and spring in years of high GMA. Systolic, diastolic blood pressure (SBP, DBP) and heart rate (HR) were measured. Pulse pressure (PP) was calculated. Data for some subjective psycho-physiological complaints (SPPC) were gathered also (Dimitrova 2008).

Hourly data about CRI from Rome neutron monitor, were used. Data about GMA, estimated by hourly Dst-index were got from WDC, Kyoto. Fig. 1a,b show hourly CRI and hourly Dst-index variations for the both periods of examinations. Table 1 presents the number of physiological measurements, which were accomplished for the different percents (levels) of CRI decreases. GMA was divided into five levels taking into account Dst-index values (Table 2).

Aalysis of variance (ANOVA), Post-hoc and the method of superimposed epochs were used to study the effect of CRI and GMA up to 3 days before and 3 days after their variations on the physiological parameters.

Table 1. CRI decrease in percents and the number of measurements

\begin{tabular}{llllllll}
\hline CRI, $\%$ & 3 & 4 & 5 & 6 & 7 & 8 & 9 \\
\hline Meas. & 194 & 715 & 930 & 627 & 253 & 39 & 41 \\
\hline
\end{tabular}


Table 2. Dst-index levels and the number of measurements

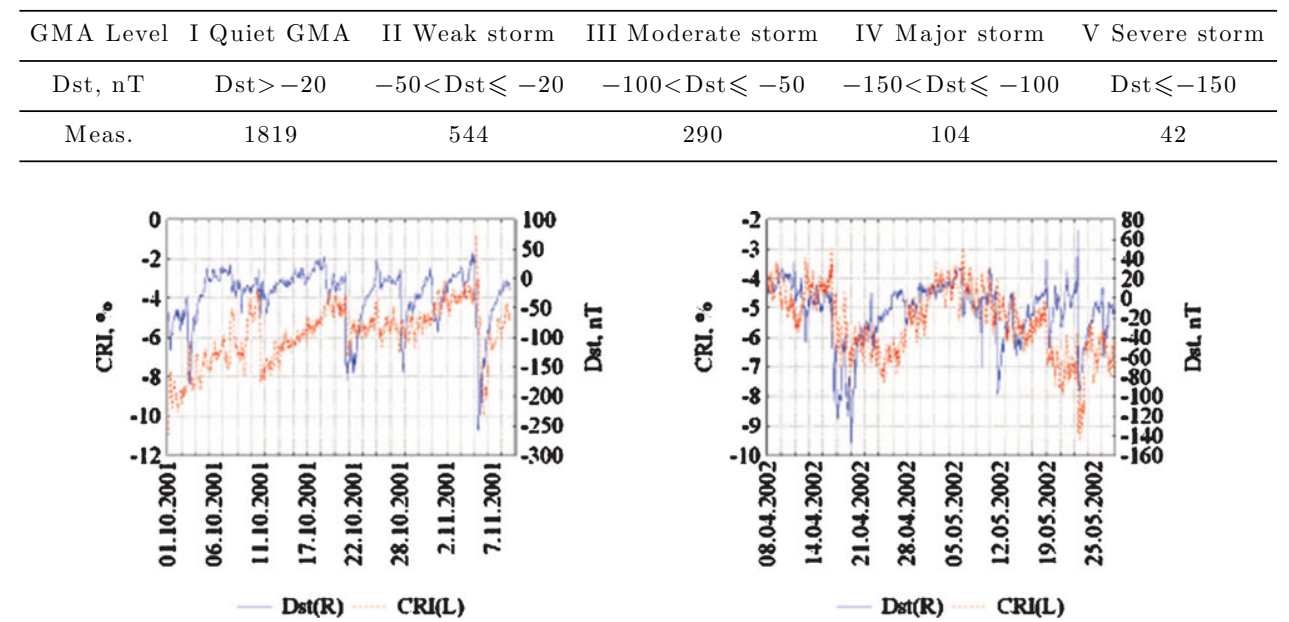

Figure 1. a) Hourly CRI and Dst-index variations during autumn examination period. b) Hourly CRI and Dst-index variations during spring examination period.

Table 3. Significance level p of CRI and GMA variations effects on the physiological parameters; * denotes statistically significant effect.

\begin{tabular}{cccccc}
\hline Factor & SBP & DBP & PP & HR & SPPC \\
\hline CRI & $0.000^{*}$ & $0.000^{*}$ & $0.010^{*}$ & 0.152 & $0.002^{*}$ \\
\hline GMA & $0.000^{*}$ & $0.000^{*}$ & $0.003^{*}$ & 0.719 & $0.000^{*}$ \\
\hline
\end{tabular}

\section{Results}

CRI variations and physiological parameters. ANOVA revealed statistically significant effect for CRI on SBP, DBP, PP and SPPC, Table 3. Fig. 2a shows the mean values of SBP and DBP for the group under different CRI decreases: SBP and DBP increased with the decrease of CRI. The maximal increment for SBP was $10.5 \%$ and for DBP $11.4 \%$. Post hoc analyses established that SBP and DBP were significantly higher during CRI decrease with $8 \%$ and $9 \%$ in comparison to CRI decrease with $3 \div 7 \%$.

PP increased also with CRI decrease, having the highest value ( $10 \%$ increment) at $8 \% \mathrm{CRI}$ decrease. The largest variation for HR was only $2.8 \%$. SPPC increased with CRI decrease and $26.8 \%$ of the persons reported SPPC during CRI decrease with $9 \%$.

Statistically significant effect on SBP and DBP (Fig. 2b) from -3rd till +3 rd day of different CRI decreases was obtained. It was revealed by Post hoc analyses that arterial blood pressure $(\mathrm{ABP})$ mean values increased significantly from -1 st till +3 rd day when CRI decreased with 8-9\%. ABP was high also on the days before, during and after CRI decrease of $7 \%$.

PP was statistically significantly affected not only on 0 day but also on +1 st and +2 nd day. PP's mean values of the group were highest from -1 st to +3 rd day when CRI decreased with $8 \%$ and on $-3 \mathrm{rd}$ and $+3 \mathrm{rd}$ day of CRI decrease with $9 \%$. SPPC increased statistically significantly on $0,+1$ st, and +3 rd day. Reported subjective complaints in the group were largest from -1 st to +3 rd day of CRI decrease with $9 \%$.

GMA variations and physiological parameters. GMA effect on SBP, DBP, PP and SPPC was statistically significant, Table 3. GMA increase was followed by an increase of the physiological parameters and the range of changes for SBP and DBP (Fig. 3a), PP and SPPC were respectively $10.7 \%, 10.4 \%, 11.2 \%$ and $21.3 \%$. Post hoc analyses revealed that the group increased significantly ABP still at moderate storms. The number of the persons who reported SPPC increased significantly still at major storms in comparison with quiet GMA and weak storms. 

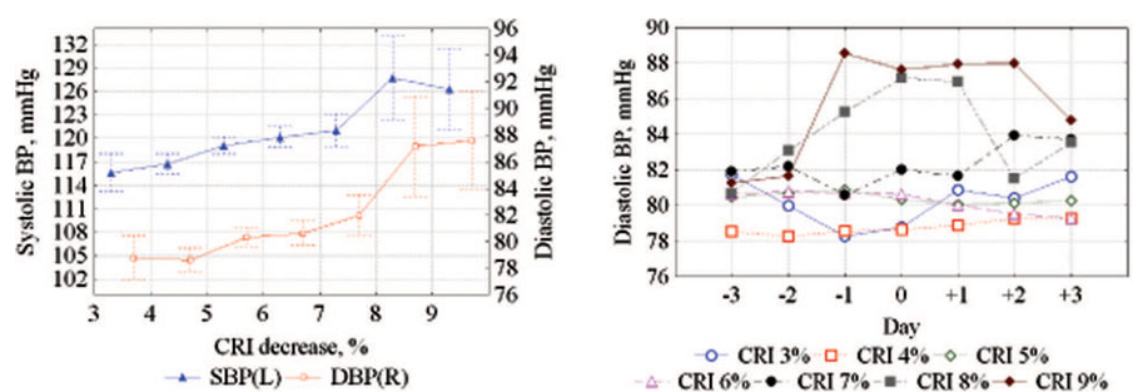

Figure 2. a) CRI effect on SBP and DBP $( \pm 95 \%$ CI $)$ b) CRI effect on DBP before, during and after CRI decreases.
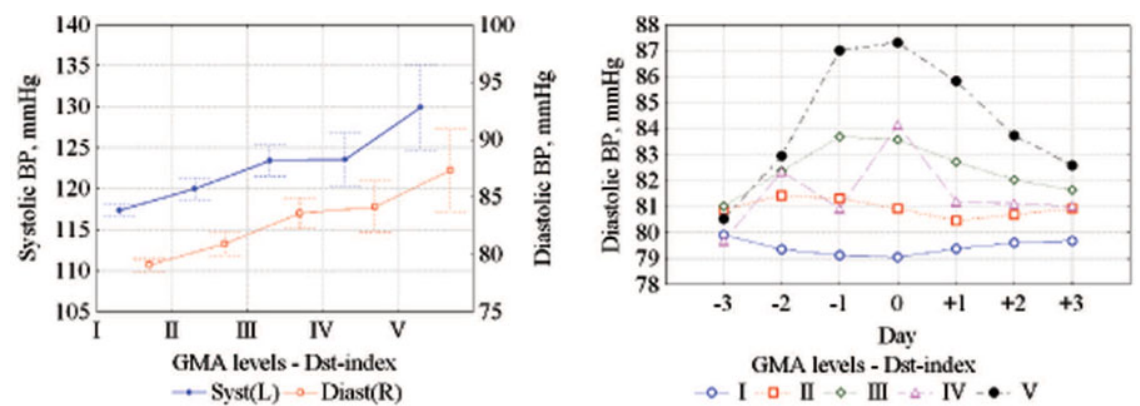

Figure 3. a) GMA effect on SBP and DBP $( \pm 95 \%$ CI $)$ b) GMA effect on DBP before, during and after geomagnetic storms.

HR of the group increased with GMA increment but with only $1.9 \%$. It was established that SBP and DBP (Fig. 3b) increased statistically significantly from -2 nd till +3 rd day, PP from -1 st till +1 st day and SPPC from -1 st till 0 day.

\section{Conclusions}

Both space weather parameters (CRI and Dst-index ) were related to statistically significant changes in the human physiological state of the examined group. It was established that SBP, DBP, PP and SPPC of the healthy volunteers increased with CRI decrease and GMA increase and on the days before, during and after their variations.

$\mathrm{ABP}$ values of the group were highest from -1 st till +3 rd day when CRI decreased more than $7 \%$ and from -2 nd to +3 rd of moderate, major and severe geomagnetic storms.Reported SPPC increased the most from -1 st till +3 rd day of the largest decreases in CRI and from -1 st till 0 day of different geomagnetic storms.

The fact that the group increased ABP on average with about $10-11 \%$ and almost $1 / 3$ from the persons felt some psycho-physiological discomfort deserves attention from a medical point of view and enhance biological, clinical and social importance of the influences examined.

\section{Acknowledgment}

This work was partially supported by National Science Fund of Bulgaria under contract NIP L-1530/05. SVIRCO NM is supported by IFSI/INAF-UNIRoma3 Collaboration.

\section{References}

Dimitrova, S. 2008, JASTP, 70/2-4, 420

Dorman, L. I 2005, Annales Geophysicae, 23, 2997

Ptitsyna, N. G., Villoresi, G., Dorman, L. I., Iucci, N., \& Tiasto, M. I. 1998, UFN, 168 (7), 767

Villoresi, G., Dorman, L. I., Ptitsyna, N. G., Iucci, N., \& Tiasto, M. I. 1995, 24th ICRC, 4, 1106 